\title{
Crystallization of Amorphous Silicon Thin Film by Using a Thermal Plasma Jet
}

\author{
Hyun Seok Lee, Sooseok Choi, Sung Woo Kim, and Sang Hee Hong* \\ Department of Nuclear Engineering, Seoul National University \\ Seoul 151-742, Republic of Korea \\ *Corresponding author: hongsh@snu.ac.kr
}

\begin{abstract}
Amorphous silicon (a-Si) films deposited on glass substrates were crystallized using a thermal plasma jet and the treated films are analyzed to find the relationship between plasma characteristics and crystallization process conditions. The crystallization process conditions were found to have different optimal operating regimes depending on the nozzle geometry. Numerical analysis of the thermal plasma jets showed that the different operating regimes for crystallization were caused by modifications of the plasma characteristics by the nozzle geometry. It is revealed that a stepped-divergent nozzle is more efficient for the thermal plasma annealing process than the conventional cylindrical one due to the broadened high-temperature region and the lowered axial velocity in the plasma jet.
\end{abstract}

Keywords : Crystallization; Polycrystalline silicon; Thermal plasma annealing; Anode nozzle geometry; 


\section{Introduction}

Recently, the demand for polycrystalline silicon (poly-Si) films has increased in the field of large-area TFT-LCD (Thin-Film Transistor-Liquid Crystal Display) and thin-film solar cells. Excimer laser annealing (ELA) is used most widely for the crystallization of amorphous $\mathrm{Si}(\mathrm{a}-\mathrm{Si})$ films but it has some disadvantages, such as narrow crystallization region and high process cost. For this reason, various alternative crystallization processes have been investigated as substitutes for this ELA technique [1-3].

Thermal plasma annealing has the potential to become an alternative crystallization method for a-Si films because the thermal plasma system has some advantages in annealing processes, such as the simple structure of the equipment, availability of atmospheric pressure process, flexible control of the power density and lower processing cost than ELA. There are several reports on thermal plasma annealing on a-Si films in these days. However, these studies focused mainly on the material characteristics of the films treated by thermal plasma jets. There are no reports on the relationship between the thermal plasma characteristics and crystallization process nor have there been any attempts aimed at modifying and analyzing the characteristics of thermal plasma jets to improve the efficiency of the annealing process [4-6].

In this study, thermal plasma jets were used as a heat source to crystallize a-Si films deposited on glass substrates, and the relationship between the thermal plasma characteristics and crystallization processing conditions were investigated experimentally and numerically. In order to control the characteristics of the thermal plasma jet generated by a DC plasma torch, two types of anode nozzle were considered and compared in terms 
of the temperature and axial velocity of the thermal plasma jet produced. A twodimensional numerical simulation was used to predict the tendency of the variations in plasma characteristics depending on the nozzle geometry. The calculated results were used to interpret the optimal processing regimes of the crystallization conditions determined by material analyses of the treated films.

\section{Experimental Procedure}

A schematic diagram of the thermal plasma annealing system is shown in Fig. 1. The thermal plasma jet from a non-transferred DC plasma torch, which is mounted on the transport device, scans the a-Si films on a glass substrate fixed to an aluminum plate with a scanning speed ranging from $0 \mathrm{~mm} / \mathrm{s}$ to $200 \mathrm{~mm} / \mathrm{s}$. The thermal plasma jet is generated by arc discharge between the tungsten cathode with a conical shape and the copper anode with a nozzle shape, and both electrodes are water cooled to prevent thermal damage. The plasma torch was operated with DC input powers of 6 to $15 \mathrm{~kW}$ and argon flow rates of 30 to $35 \mathrm{lpm}$, and the distance between the anode nozzle exit and film was set to $15 \mathrm{~mm}$. 500 nm-thick a-Si films were deposited on $\mathrm{SiO}_{2}(300 \mathrm{~nm}) /$ glass (Corning $®$ Eagle $\mathrm{XG}^{\mathrm{TM}}$ ) substrates by low pressure chemical vapor deposition (LPCVD). The coated substrates were then cut to a width of $30 \mathrm{~mm}$ for the crystallization experiments. Figure 2 shows the geometry of a conventional cylindrical nozzle and a new stepped-divergent nozzle considered in this study. The stepped-divergent nozzle was used to control plasma characteristics and improve the efficiency of thermal plasma annealing process. In the previous study on the nozzle geometry, arc shunting is known to be restricted due to flow 
separation next to the nozzle step, which reduces the voltage fluctuations while operating the torch [7]. Furthermore, the high-temperature central region of the thermal plasma jet is diffused outwards and expanded in the direction of the radius along the divergent region in the stepped-divergent nozzle.

The crystallinity of the treated films was evaluated by Raman scattering spectroscopy. In this evaluation, an Ar laser with a $514 \mathrm{~nm}$ wavelength was used as the excitation light with an exposure time of $25 \mathrm{~s}$. The center of the process window was selected as the measurement point.

\section{Numerical modeling}

The thermal plasma characteristics generated under the crystallization process conditions for the torch input power and scanning speed are calculated by numerical modeling for both types of anode nozzle. Two numerical modeling steps are performed to determine the thermodynamic flow on the substrate surface during the thermal plasma annealing process. First, the arc discharge inside the torch is analyzed using a twodimensional numerical code, DCPTUN (DC Plasma Torch in Unstructured Grid System), which was developed previously in the authors' laboratory [8]. The validity of the numerical process is confirmed by comparing the experimental input power with the calculated thermal plasma power considering the torch efficiency. Through this numerical modeling inside the plasma torch, the plasma characteristics calculated at the nozzle exit are regarded as the inlet boundary conditions for the next numerical modeling outside the torch, and the thermal plasma jet flow ejected from the torch exit is simulated using the 
commercial Fluent ${ }^{\circledR}$ code. Since both the numerical simulations are performed under an assumption of steady-state thermal flow, the influence of the annealing duration according to the scanning speed is not taken into account. Although the annealing duration and heat transfer between the thermal plasma jet and films are not considered in this numerical work, the calculated numerical results of the plasma characteristics are still reasonable. This is because the amount of heat transferred toward the substrate is much smaller than that of the total thermal energy in the thermal plasma jet.

\section{Results and discussions}

The crystallization process conditions are found by observing and analyzing the treated films, as shown in Fig. 3 for input power to the torch and scanning speed under a constant nozzle-to-film distance of $15 \mathrm{~mm}$. The input current to the torch with the cylindrical nozzle is $300 \mathrm{~A}$ for an average arc voltage of $30 \mathrm{~V}$, which corresponds to an input power of approximately $9 \mathrm{~kW}$. On the other hand, the input currents with the stepped-divergent nozzle range from 400 to 440 A corresponding to torch powers from 12 to $13.5 \mathrm{~kW}$. The data points marked with $\square$ and $\bigcirc$ show the crystallization process conditions for the stepped-divergent nozzle and cylindrical nozzle, respectively, as determined from Raman scattering spectroscopy. In the right-hand-side processing regime with higher scanning speeds and relatively low input power from the marked regime, the a-Si films treated with the respective thermal plasma were not crystallized and remained amorphous. However, the treated films were damaged in the left-hand-side regime with lower scanning speeds and relatively high input power. These three distinct regimes suggest that there are proper 
operating conditions for the stable annealing of a-Si films by thermal plasma treatment depending on the torch nozzle type, as shown in the diagram. Hence, the thermal plasma characteristics, which eventually determine the maximum temperature and cooling rate of the films during annealing, can be controlled effectively by the nozzle geometry.

The influence of the nozzle geometry on the plasma characteristics was examined by numerical simulation results, as shown in Fig. 4 for the same operating current of 300 A but with different nozzle structures under a constant nozzle-to-film distance of $15 \mathrm{~mm}$. From the two-dimensional distributions in the plasma jet region from the anode nozzle exit to the film surface, the stepped-divergent nozzle yields a broader high-temperature region and much lower axial velocities than the cylindrical nozzle. As indicated in Fig. 3, an input current of $300 \mathrm{~A}$, i.e., an input power of $9 \mathrm{~kW}$, is not suitable for crystallization in the stepped-divergent nozzle, which is in contrast to the cylindrical nozzle. In view of the reduced influence of the axial velocity on the film temperature and its gradient, it is believed that the temperature of the plasma jet plays an important role in the crystallization process.

On the other hand, the numerical results according to different operating currents and flow rates are shown in Fig. 5 for corresponding input powers of $9 \mathrm{~kW}$ and $12 \mathrm{~kW}$ to the cylindrical nozzle and stepped-divergent nozzle, respectively, which are both the proper crystallization process conditions with a scanning speed of $80 \mathrm{~mm} / \mathrm{s}$ and a nozzle-to-film distance of $15 \mathrm{~mm}$. It is seen that the temperature profiles near the film surface are similar for the two nozzle cases despite the different input powers. Therefore, considering the previous results in Fig. 4, a maximum temperature of approximately $10000 \mathrm{~K}$ in the central region of the thermal plasma jet is needed to crystallize the a-Si films under these process 
conditions. It should be noted that the high temperature region $>9000 \mathrm{~K}$ is broader and the axial velocities are still lower for the stepped-divergent nozzle compared with the cylindrical nozzle. From the experimental results, the average width of the crystallized area on the treated film is estimated to be up to $10 \mathrm{~mm}$ when the stepped-divergent nozzle is used while it is approximately $5 \mathrm{~mm}$ when the cylindrical one is used, even though the former requires approximately $30 \%$ more input power than the latter. In conclusion, the thermal plasma using the stepped-divergent nozzle turns out to improve the overall power efficiency of the annealing process.

Figure 6 shows the Raman scattering spectra of the crystallized films marked in Fig. 3. In addition, the crystallization fractions of these films are listed in Table 1. The crystallization fraction, which is defined by $X_{c}=\left(I_{c}+I_{m}\right) /\left(I_{c}+I_{m}+I_{a}\right)$, can be calculated from the crystalline $\left(I_{c}\left(\right.\right.$ at $\left.\sim 520 \mathrm{~cm}^{-1}\right)$, intermediate $\left(I_{m}\left(\right.\right.$ at $\left.\sim 500 \mathrm{~cm}^{-1}\right)$ and amorphous $\left(I_{a}\left(\right.\right.$ at $\left.\sim 480 \mathrm{~cm}^{-1}\right)$ Gaussian peaks in the Raman scattering spectrum [9]. The TO phonon peaks appear at 517 to $519 \mathrm{~cm}^{-1}$ corresponding to the poly-Si phonon peak. By increasing the input power and decreasing the scanning speed, the TO phonon peaks move toward $520 \mathrm{~cm}^{-1}$ and the crystallization fraction increases. This indicates that efficient heat flux to the film surface enhances the crystallization of a-Si films, and the perfect melting of a-Si films is an ideal phase for producing highly crystallized poly-Si films. In addition, it is observed that the crystallization fraction is reduced, when the input power is higher than a certain level, corresponding to the current value of $440 \mathrm{~A}$, as appeared in Table 1. Therefore, it is thought that there exists an optimum value of input power for crystallization between $420 \mathrm{~A}$ and $440 \mathrm{~A}$. 


\section{Conclusion}

In this thermal plasma process, 500-nm-thick a-Si films were deposited on glass substrates, and annealed and crystallized using a plasma torch with two different anode nozzle geometries to modify the thermal plasma characteristics. The stepped-divergent and the cylindrical nozzles of the torch were used to control the plasma jet, and the crystallization process conditions were varied according to the nozzle geometry. The influence of the nozzle geometry on the thermal plasma characteristics were analyzed by numerical modeling of the plasma jet. The results showed that the stepped-divergent nozzle can expand the high-temperature central region and reduce the axial velocity of the plasma jet. These effects of stepped-divergent nozzle are expected to enhance the efficiency of the crystallization process using the plasma jet. In the experimental results, the crystallization process window for the torch input power and scanning speed with the stepped-divergent nozzle is almost double that with the conventional nozzle. In addition, it is revealed that the efficiency of the process can be eventually enhanced by the simple modification of the nozzle geometry. The crystallinity of the treated films increases when sufficient heat flux is supplied to the a-Si films but decreases when the film surface is exposed to excessive heat. The treated films with a crystallinity $>90 \%$ can be produced by optimizing the thermal plasma process, which can compete with that of ELA treated films [9]. Although there is difficulty in achieving uniform crystallization with the thermal plasma annealing method, this method is still attractive because it can easily produce highly crystallized films with lower processing costs than the conventional ELA method. 


\section{Acknowledgment}

This work was supported by the Ministry of Knowledge Economy through the Regional Innovation Center for Environmental Technology of Thermal Plasma (ETTP) at Inha University.

\section{References}

[1] C. C. Kuo, W. C. Yeh, J. F. Lee, J. Y. Jeng, Thin Solid Films 515 (2007) 8094.

[2] W. E. Hong, J. S. Ro, Thin Solid Films 515 (2007) 5357.

[3] M. M. A. Hakim, I. Matko, B. Chenevier, P. Ashburn, Microelectron. Eng. 83 (2006) 2437.

[4] S. Higashi, H. Kaku, T. Okada, H. Murakami, S. Miyazaki, Jpn. J. Appl. Phys. 45 (2006) 4314.

[5] T. Okada, S. Higashi, H. Kaku, T. Yorimoto, H. Murakami, S. Miyazaki, Solid-State Electron. 52 (2008) 377.

[6] K. Haruta, M. Ye, Y. Takemura, T. Kobayashi, T. Ishikawa, J. K. Saha, H. Shirai, NonCryst. Solids 354 (2008) 2333.

[7] S. Choi, T. H. Hwang, J. H. Seo, D. U. Kim, S. H. Hong, IEEE T. Plasma Sci. 32 (2004) 473.

[8] M. Hur, K. S. Kim, S. H. Hong, Plasma Sources Sci. Technol. 12 (2003) 255.

[9] C. Yi, S. W. Rhee, J. H. Ju, S. K. Yim, H. Min, J. Mater. Sci. : Mater. Electron. 12 (2001) 697 


\section{List of table caption}

Table 1

Crystallinity of the poly-Si films treated by the thermal plasma annealing under various process conditions for current corresponding to input power and scanning speed.

\section{List of figure caption}

Fig. 1

Schematic diagram of a thermal plasma annealing system, the DC plasma torch is transversely movable by a transport device to scan the films on a glass substrate.

Fig. 2

Geometry of a conventional cylindrical nozzle and a newly designed stepped-divergent nozzle considered in this study.

Fig. 3

Optimal regimes of the crystallization processing conditions for the torch input power and scanning speed, which were determined from the Raman scattering spectra of the films treated by the thermal plasma annealing using the two different types of anode nozzle. 
Fig. 4

Two-dimensional distributions of the calculated (a) temperature and (b) axial velocity of the thermal plasma jets produced by the stepped-divergent nozzle (left side of figure) and cylindrical nozzle (right side of figure), operated with the same current (300 A) and flow rate $(30 \mathrm{lpm})$.

Fig. 5

Two-dimensional distributions of the calculated (a) temperature and (b) axial velocity of the thermal plasma jets produced by the stepped-divergent nozzle (left side of figure) operated with a current of $400 \mathrm{~A}$ and a flow rate of $35 \mathrm{lpm}$, and by the cylindrical nozzle (right side of figure) operated with $300 \mathrm{~A}$ and $30 \mathrm{lpm}$.

Fig. 6

Comparison of the Raman scattering spectra of the films crystallized by thermal plasma annealing using the two types of nozzle operated at different input powers and scanning speeds. 
Table 1

\begin{tabular}{c|cccc|ccc}
\hline Nozzle Type & \multicolumn{3}{c|}{ Stepped-Divergent Nozzle } & \multicolumn{3}{c}{ Cylindrical Nozzle } \\
\hline Current (A) & 400 & 400 & 420 & 440 & 300 & 300 & 300 \\
\hline $\begin{array}{c}\text { Scanning Speed } \\
(\mathrm{mm} / \mathrm{s})\end{array}$ & 70 & 80 & 80 & 80 & 70 & 80 & 90 \\
\hline $\begin{array}{c}\text { Crystallization } \\
\text { Fraction (\%) }\end{array}$ & 92.1 & 91.8 & 94.4 & 92.3 & 94.6 & 92.5 & 89.3 \\
\hline
\end{tabular}




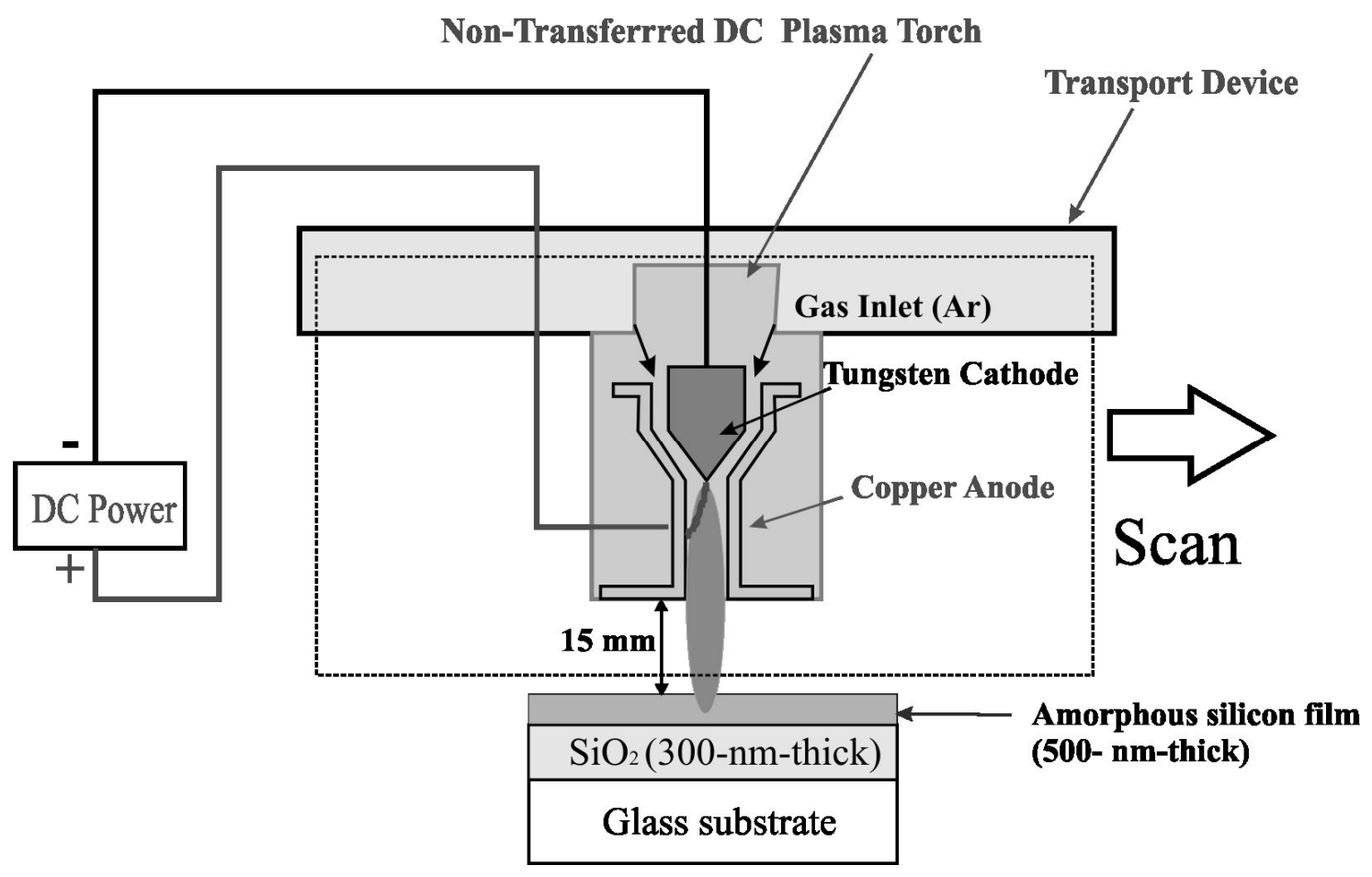

Fig. 1 

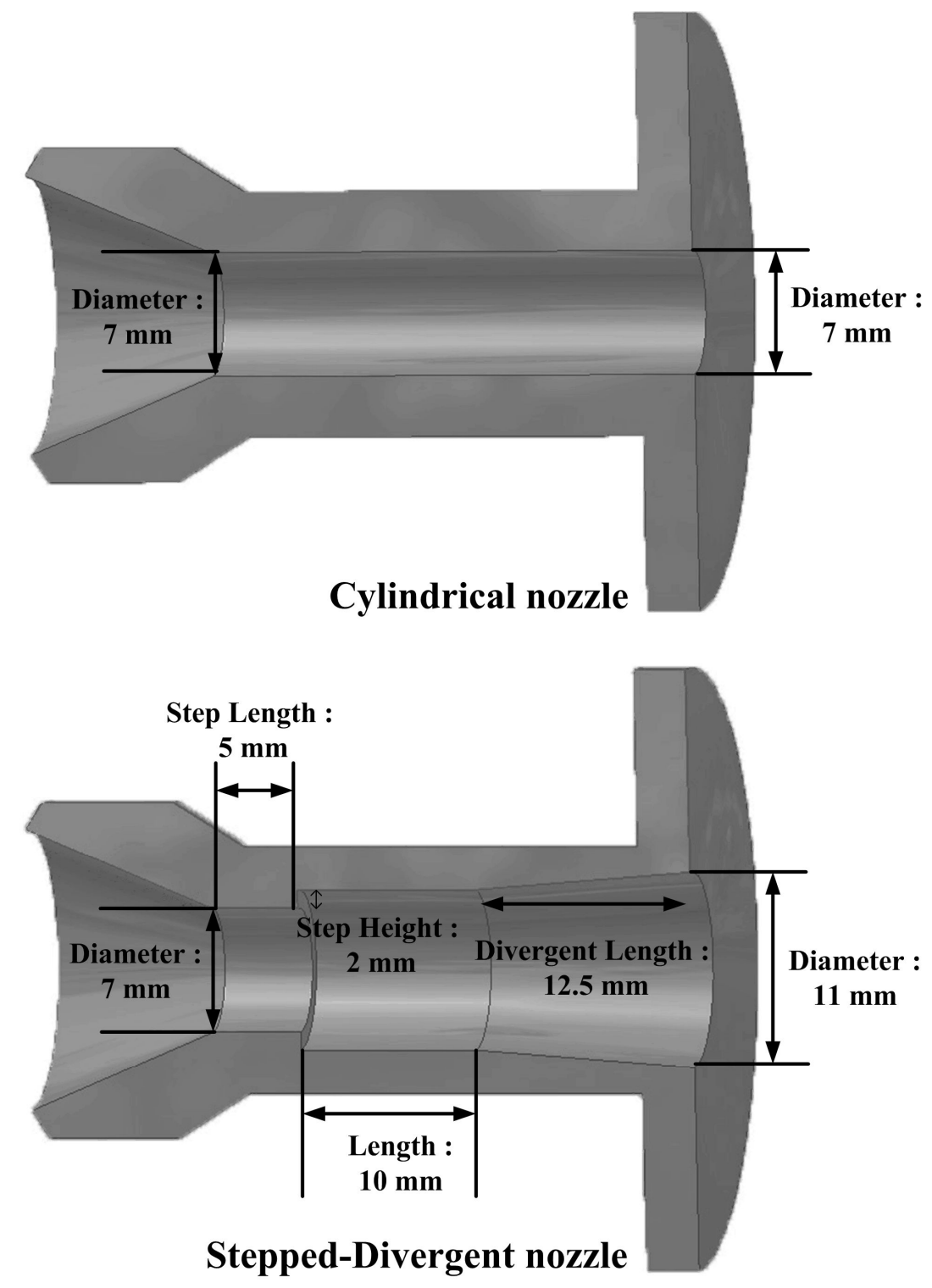

Fig. 2 


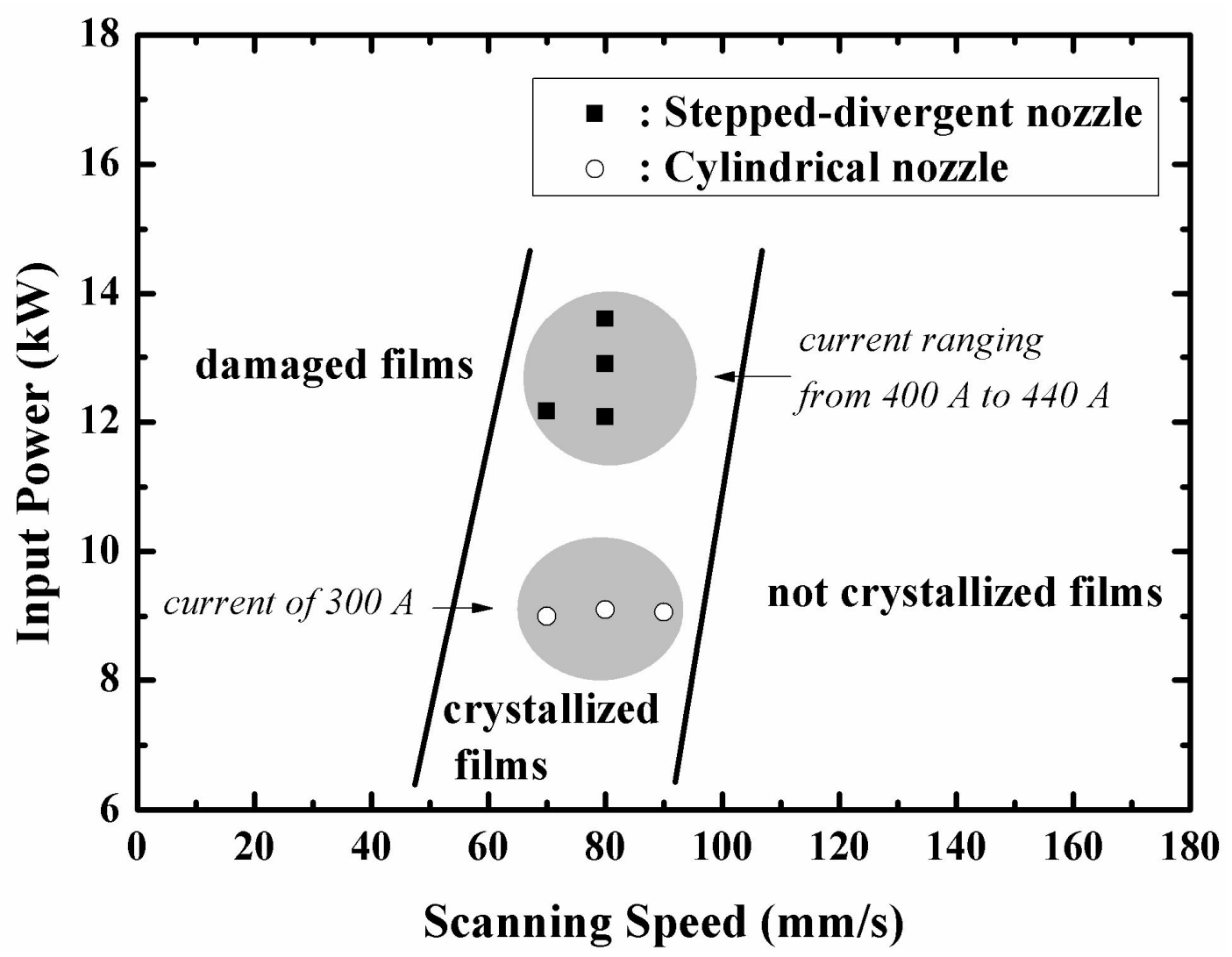

Fig. 3 


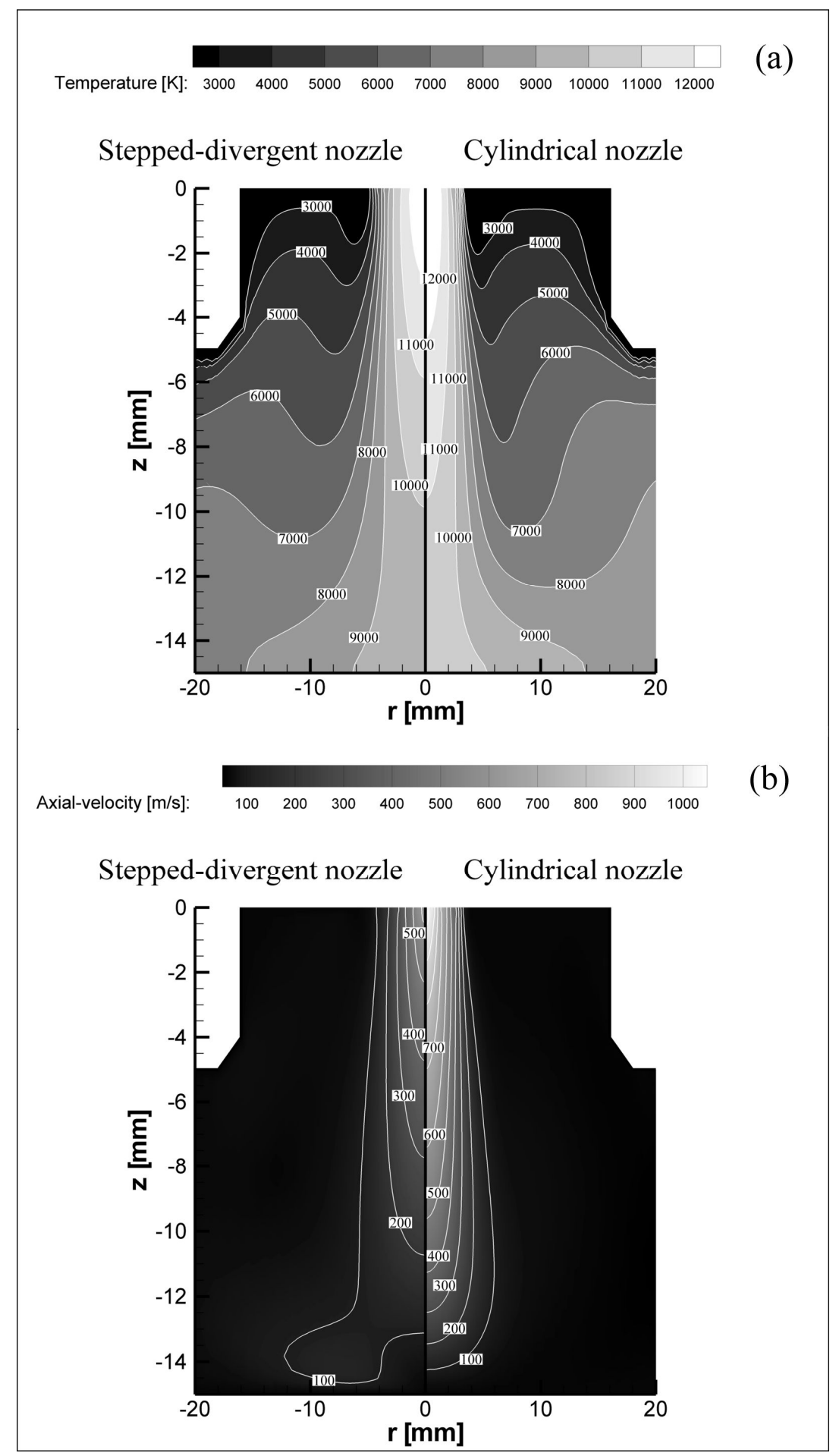

Fig. 4 


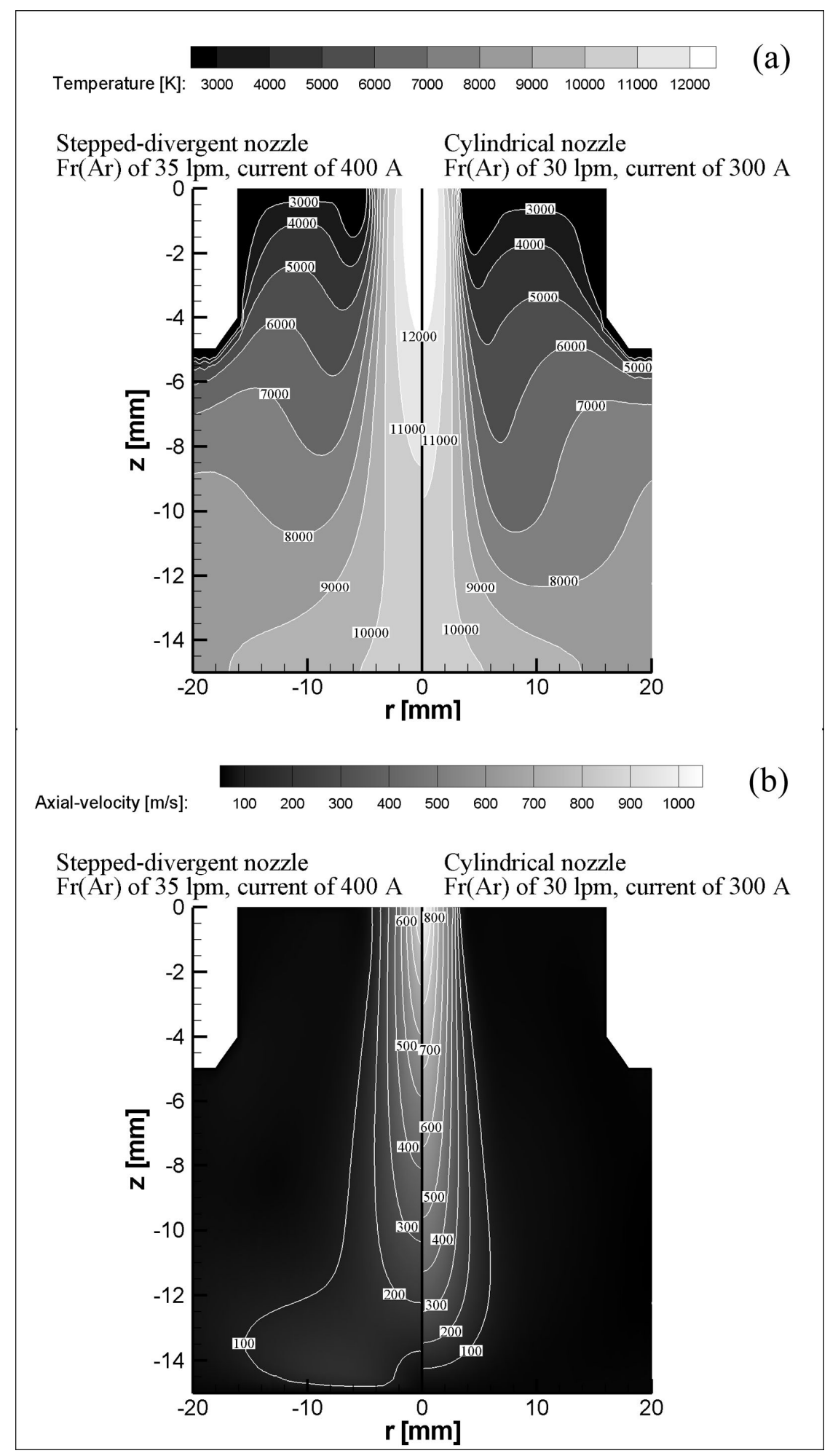

Fig. 5 


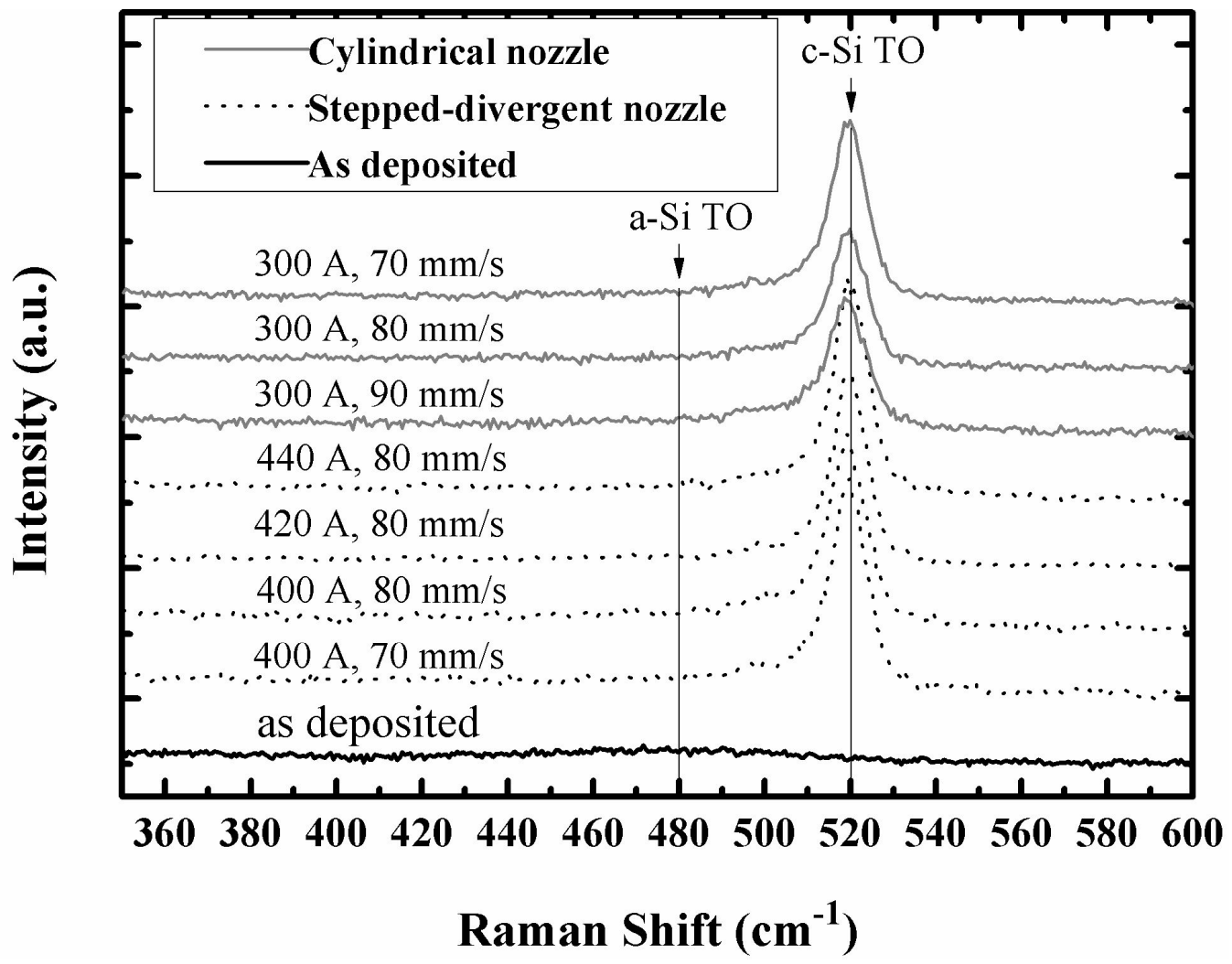

Fig. 6 